\title{
LA LUTTE CONTRE LA CORRUPTION DANS LE SYSTEME INTERAMERICAIN DE PROTECTION DES DROITS DE L'HOMME
}

THE COMBAT OF THE CORRUPTION IN THE INTERAMERICAN SYSTEM OF HUMAN RIGHTS

George Sarmento

Post-doctorat à l'Université d'Aix-Marseille. Chercheur au Laboratoire des droits de l'homme/UFAL. Professeur à l'Université Fedérale d'Alagoas. Professeur invité à l'Université de Montpellier1 et d'Aix-Marseille. Doctorat en Droit à l'Université Fédérale de Pernambuco. Procureur au Ministère Public de l'État d'Alagoas, Brésil. george_sarmento@uol.com.br Reçu: 2016-08-24. Accepté: 2016-10-10.

Résumé: Cet article vise à montrer les stratégies conçues par le Système interaméricain de protection des droits de l'homme en matière de prévention, de détection et des poursuites contre la corruption dans la gestion publique. Pour développer notre réflexion, nous allons présenter les principales approches qu'envisagent expliquer ce phénomène, ainsi que montrer l'articulation entre les principaux organismes supranationaux liées à l'Organisation des Etats Américains pour donner des réponses rationnelles et efficaces à cette forme de criminalité.

Mots-clés: Corruption - Abus de pouvoir - Gestion publique - Prévention et répression

Abstract: This article aims to show the strategies designed by the American system of protection of the human rights in the prevention, detection and action against corruption in public administration. To develop our reflection, we will present the main approaches that consider explain this, and show the relationship between the main supranational organizations linked to the Organization of American States to give rational and effective responses to such crime .

Keywords: Corruption - Abuse of power - Public management - 
Prevention and punishment

\section{INTRODUCTION}

La corruption est devenue un problème majeur non seulement dans le cadre de la politique interne des États, mais aussi dans les relations internationales. Aujourd'hui elle dépasse les frontières nationales et bouleverse l'économie mondiale. Elle déplace des sommes pharamineuses qui transitent librement dans des paradis fiscaux en profitant de la libération des échanges, des flux financiers et de la fragilité des systèmes de contrôle fiscal, administratif et judiciaire. La corruption dans les institutions gouvernementales constitue un frein aux droits sociaux, une menace pour la démocratie, un facteur de déstabilisation de l'ordre macroéconomique, une manipulation des marchés publics et un élément de renforcement de la pauvreté. Enfin, elle est à l'origine des financements occultes de partis politiques, du dysfonctionnement des services publics, de l'accroissement du crime organisé, de l'affaiblissement de la confiance que les citoyens doivent porter aux institutions démocratiques de l'État.

Le dernier rapport de l'OCDE sur la corruption transnationale, publié en décembre 2014, présente des statistiques bouleversantes. Il a analysé le résultat de 427 affaires concernant des actions répressives engagées à l'encontre des personnes physiques et morales et qui ont été conclues entre le 15 février 1999 et le $1^{\mathrm{T}}$ juin 2014. Selon le rapport, 53\% des affaires ont impliqué des versements de pots-de-vin dans le cadre des marchés publics, dont les principaux secteurs sont l'extraction, la construction, le transport et l'entreposage, l'information et la communication. Ensemble, ils représentent deux-tiers des affaires de corruption. $80 \%$ des agents publics corrompus sont des salariés des entreprises publiques, suivis d'autres fonctionnaires comme des Chefs d'État, ministres, agents de défense, élus, agents de douanes, etc. Le montant des commissions en pourcentage est assez variable, entre $21 \%$ et $2 \%$, selon le secteur d'activité 1 .

Ce phénomène, complexe et répandu, exige des réponses sévères en matière de prévention, de détection et de poursuites. Sur le plan international plusieurs initiatives ont été prises pour lutter efficacement contre la corruption. Les Nations Unies, les systèmes régionaux et des

$121 \%$ extraction, $19 \%$ commerce de gros et de détail, $17 \%$ activités de services administratifs, $16 \%$ fabrication, $14 \%$ santé humaine, $13 \%$ autres activités et services, $8 \%$ administration publique et services, $11 \%$ électricité et gaz, $8 \%$ administration publique et défense, $6 \%$ activités scientifiques et techniques. Moins de $5 \%$ : information et communication, construction, agriculture, sylviculture et pêche, éducation et distribution d'eau. Cf. Rapport de l'OCDE sur la corruption transnationale : une analyse de l'infraction de corruption. France : Éditions OCDE, 2014. 
organismes multilatéraux ont produit plusieurs documents normatifs conventions, protocoles, recommandations, par exemple - et des outils pour aider les pays dans ce combat, comme l'entraide judiciaire, le gel et la confiscation de biens.

Quels sont les circonstances qui ont favorisé la lutte contre la corruption à l'échelle internationale ? Comment s'articule le droit international pour mener ce combat de façon efficace?

\section{APROCHES SUR LA CORRUPTION}

À partir de l'approche économique (A), sociologique (B) et juridique $(\mathrm{C})$, cet article se propose d'exposer ici les principaux résultats, fondements, expectatives et mise en œuvre des mesures présentées par les principaux organismes chargés de sanctionner la corruption politique. Pour y parvenir, il faut décrire les aspects les plus importants du système international de lutte contre la corruption, y compris les actions menées par l'Organisation des États Américains. Afin de souligner la dimension préventive, répressive et dissuasive des conventions internationales et du droit interne des États parties, seront présentées quelques hypothèses d'affrontement aux atteintes à la probité publique dans le rapport à la passation des marchés publics et ses échanges illicites.

\section{A. Approche économique}

L'occupation des marchés publics ${ }^{2}$ à l'étranger par des sociétés multinationales est à l'origine de la lutte contre la corruption des agents publics. En fait, la corruption active était la règle dans la passation des marchés publics à l'étranger. Dans les années 70, plusieurs pays européens ont adopté des lois qui permettaient la déduction fiscale des sommes versées par des entreprises aux fonctionnaires étrangers. En matière de transactions internationales, les États de l'OCDE toléraient le versement de frais commerciaux exceptionnels à des agents publics en tant que dépenses légales et déductibles officiellement. C'était l'institutionnalisation du pot-de-vin dans les transactions transnationales ${ }^{3}$.

Un exemple s'impose. En 1976, la France a institué la déductibilité fiscale des frais commerciaux exceptionnels conditionnée

2 Les marchés publics sont des secteurs d'activité économique qui mouvementent d' importants chiffres d'affaires. Par exemple, pétrole, gaz, électricité, défense et armement, banques, aéronautique civile et militaire, industries, pêche, sylviculture, médicaments, santé, télécommunications, mines, immobilier, entreposage, transports, informatique, technologie de l'information, construction civile, biotechnologie, recherches scientifiques, bâtiment et des travaux publics (BTP).

3 Senat National. Projet de loi relatif à la lutte contre la corruption : La lutte contre la corruption, un cadre juridique en mouvement. Paris : Senat.fr . Consulté en 02/12/2014. 
à l'autorisation préalable des autorités douanières. Les entreprises étaient obligées de déclarer les sommes versées à des autorités étrangères pour enlever des contrats d'exportation. Par contre, elles avaient le droit d'en déduire le montant de leur bénéfice imposable. La jurisprudence administrative tolérait cette pratique, mais conditionnait le bénéfice fiscal à la preuve comptable du paiement de la commission à une personne physique ou morale - y compris un fonctionnaire public - porteuse du pouvoir de décision en matière d'achat. Malgré quelques restrictions, surtout l'abrogation de 1993, les pots-de-vin ont été tolérés dans le pays. La fin de la déductibilité n'a disparu du droit français que par la loi du 30 juin 2000, comme conséquence de la ratification de la Convention de l'OCDE (1997) ${ }^{4}$.

Le versement des frais commerciaux exceptionnels était une pratique courante dans les entreprises multinationales siégées dans des pays de l'OCDE ${ }^{5}$ C'est le produit des théories fonctionnalistes diffusées dans les années 60, selon lesquelles les pots-de-vin stimulaient la compétitivité et contournaient les exigences bureaucratiques paralysantes qui retardaient la signature des contrats avec l'administration publique. Le paiement de commissions est devenu une nécessité pour ne pas la noyer dans des procédures administratives interminables, complexes et décourageantes, dont le fonctionnaire responsable est doté d'un fort pouvoir discrétionnaire. Chez les avocats des entreprises impliquées dans de grosses affaires, la corruption est « l'huile pour dégripper les rouages, pour contourner les règlements absurdes ou des administrations vétilleuses ${ }^{6} \gg$.

Les disputes des marchés publics sont souvent féroces et déloyales. Les appels d'offre attirent vivement l'intérêt des entreprises multinationales décidées à occuper des espaces dans des secteurs économiques des pays étrangers. Si la perte d'une affaire peut affecter lourdement l'avenir d'une entreprise, elle aura aussi des effets négatifs dans la balance commerciale du pays exportateur. Le paiement d'un pourcentage à des autorités responsables des permis, des licences et des réglementations reste donc une stratégie lucrative pour les entrepreneurs qui veulent obtenir ou maintenir des portions de marchés publics ${ }^{7}$. Enfin,

4 Daniel Dommel. Face à la corruption : peut-on l'accepter ? Peut-on la prévenir ? Peut-on la combattre? Paris : Karthala, 2003, p. 70-71.

5 La Belgique, par exemple, a adopté le principe de la non-déductibilité des commissions occultes pour obtenir des marchés publics à l'étranger en 1996. Mais elle était considérée possible dans des situations dans lesquelles il y avait des pratiques concurrentielles nécessaires dans les pays concernés à un niveau considéré raisonnable par les autorités fiscales. Après la ratification de la Convention de l'OCDE, une telle conduite est devenue punissable.

6 Daniel Dommel. Face à la corruption : peut-on l'accepter ? Peut-on la prévenir ? Peut-on la combattre ? Paris : Karthala, 2003, p. 47.

7 Daniel Dommel. Face à la corruption : peut-on l'accepter? Peut-on la prévenir ? Peut-on la combattre? Paris : Karthala, 2003, p. 47. 
une imposition du capitalisme, un vecteur du développement économique.

Plusieurs auteurs se sont consacrés à la « Théorie économique de la corruption ». Certains argumentaient que la corruption n'étaient pas incompatible avec le progrès, mais plutôt une réponse du marché aux abus de l'excessive régulation des activités économiques, surtout dans la passation des marchés publics. C'est le cas de Nathaniel Leff (1964), J. Nye (1967) et Samuel P. Huntington (1968), qui sont les principaux représentants d'une branche nommé théorie fonctionnelle de la corruption.

Selon cette conception, l'échange financier entre les entreprises et les fonctionnaires dans des transactions transnationales apportaient des avantages économiques et sociaux comme, par exemple, le développement des pays concernés et l'augmentation des emplois dans le secteur privé. Dans un texte très connu, Econonomic Development Through Bureaucratic Corruption ${ }^{8}$, paru en 1964, Nathaniel Leff constatait que la corruption était un important instrument de protection des entrepreneurs contre l'hostile interférence de la bureaucratie paralysante sur les activités économiques. Le versement de commissions à des agents publics était une réponse du marché aux entraves et à l'abus d'autorité qui troublaient les affaires des sociétés multinationales. Parmi les objectifs envisagés, la théorie fonctionnaliste mentionnait la réduction des incertitudes et du risque des ennuis administratifs dans l'analyse des projets.

En conséquence, le dessous de table n'était qu'une forme de protection des investissements dans des pays étrangers et de réduction du temps d'attente d'une demande. Paradoxalement, la relation était basée sur la confiance mutuelle et ancrée sur un contrat informel entre la société et l'agent public décideur. C'est ce que Leys (1965) appelait " corruption honnête ». Cette formulation théorique ne s'arrête pas aux années 60 . L'analyse de la corruption selon cette perspective connaît d'autres approches comme ceux de Francis Lui (1985), Pranab Bardhan (1997) et Daniel Kaufmann (1997).

Il convient de remarquer que l'analyse économique ne se consacre pas à la question de l'éthique, considérée comme objet de recherche de la philosophie morale. L'homo oeconomicus amoral prend ses décisions sur la base de facteurs rationnels. Selon Rudy Aeroudt, « son seul mobile est de déterminer son comportement en fonction de la perspective de satisfaire au mieux ses besoins. Ses décisions, nous dit l'économiste, sont prises de façon à préserver constamment un équilibre entre son effort et l'utilité marginale dégagée ${ }^{9} »$. C'est la raison pour laquelle les auteurs ne se consacraient qu'aux effets de la corruption sur les intérêts des entreprises.

Jacques Borricand rappelle que les « actes de corruption ont été

8 The Americal Behavioural Scientist, 1964, pp. 84-12.

9 Rudy Aernoudt. Corruption à Foison - Regards sur un phénomène tentaculaire. Paris :

L'Harmattan, 2003, p.21. 
trop longtemps considérés comme des faits relevant d'une criminalité sans victime $»$. Autrement dit, ils n'attiraient pas l'attention du droit pénal. Il y avait tout de même une certaine tolérance de la société vis-à-vis de certaines pratiques corrompues. Il n'est pas inutile de citer la célèbre classification proposée par Arnold Heidenheimer pour expliquer la perception de ce phénomène par la population. Il établit la distinction entre trois espèces de corruption : blanche, grise et noire, selon le dégrée de tolérance de l'opinion publique et des élites. La première est considérée comme une pratique acceptée, voire inhérente à l'activité politique ; la deuxième n'est condamnée que par une partie de la population, tandis que la troisième désigne les actes condamnés à la fois par la population et par les élites. ${ }^{10}$.

La relativisation de la corruption a toujours été présente dans le débat politique. Même aujourd'hui, le niveau de tolérance des électeurs par rapport aux élus est préoccupant. Pierre Lascoumes explique ce phénomène en présentant trois facteurs objectifs : « d'abord, ce qui fait corruption pour les uns ne le fait pas pour les autres. Les significations données à ce terme sont très diverses. Ensuite, cette forme de déviance bénéficie d'un vaste ensemble de justifications et d'excuses qui en relativisent la gravité. Enfin, il existe des liens étroits entre le type de perception de la corruption et de la conception du politique ${ }^{11} »$. Ces éléments sont de véritables obstacles à la mise en place d'une politique criminelle anticorruption efficace. On peut affirmer qu'il y a un parallélisme entre le jugement politique et le jugement judiciaire, dans la mesure où la majorité des électeurs ne considèrent pas la corruption comme une composante du crime organisé. La lenteur des poursuites judiciaires favorisent la présence des prévenus dans le paysage politique, jouant parfois le rôle de victimes d'un système juridique arbitraire ou du « gouvernement des juges », voire des manœuvres des adversaires ou d'un piège ${ }^{12}$.

\section{B. Approche rationaliste}

Sous l'influence de Max Weber et d'autres chercheurs, surtout des économistes, on a vu l'épanouissement d'une autre conception théorique : la rationalisation administrative. Parmi les défenseurs de cette approche, il convient de citer Pontes de Miranda, juriste brésilien d'orientation positiviste. Grosso modo, les auteurs proposaient la professionnalisation de la gestion publique, la formation des fonctionnaires publics, le renforcement des systèmes de contrôle interne

10 Jacques Borricand. La corruption dans l'entreprise. In : Morale Économique - des fondements religieux à l'éthique en entreprise. Aix-en-Provence : Librairie de l'Université d'Aix-en-Provence, 1999, p. 217.

11 Pierre Lascoumes. Une démocratie corruptible - arrangements, favoritisme et conflits d'intérêts. Paris : Seuil, 2011, p. 17.

12 Idem, 63. 
et externe, la réglementation des marchés publics, la quête de critères scientifiques concernant les décisions administratives, etc. Ce modèle confiait aux technocrates la mission de prendre des mesures techniques à l'occasion des appels d'offre et la planification des dépenses publiques. Dans les années 50, la technocratie était une réponse à l'empirisme, au despotisme, au clientélisme, au trafic d'influence et au favoritisme très présents dans l'action politique. Les résultats ont été faibles, insatisfaisants. D'abord à cause de la proximité entre agents publics et entreprises privées ; ensuite, le manque de légitimité démocratique et institutionnelle pour prendre des décisions importantes ; finalement, la méfiance de la classe politique envers les experts a été un facteur générateur de conflits d'intérêts, voire d' arrangements nuisibles à l'Etat. En conséquence, le rationalisme administratif tout court s'est montré incapable d'éliminer la corruption dans les marchés publics.

\section{L'approche répressive de lutte contre la corruption à l'échelle mondiale}

Les troubles à la concurrence mondiale dans les appels d'offre de grande envergure ont affecté profondément les entreprises nordaméricaines. Le manque de loyauté et la corruption endémique dans la gestion publique étaient les principaux problèmes à affronter pour qu'elles reprennent un espace important dans les marchés publics dans plusieurs continents. En 1977, les États-Unis ont adopté le Foreign Corrupt Practices Act, une loi qui sanctionnait sévèrement l'offre de corruption (supply side) par des fonctionnaires étrangers. Cette loi a été élaborée en conséquence de l'affaire Whatergate, qui a écarté le président Nixon du pouvoir. Les enquêtes menées par la police judiciaire ont présenté les preuves irréfutables de la participation des entreprises américaines au versement de pots-de-vin à des agents publics étrangers pour obtenir le contrôle des marchés publics. Mais le scandale le plus célèbre a été l'affaire Lockheed Corporation, société accusée de verser U\$ 25 millions à des autorités japonaises, chargées de l'achat des avions Tristar L-1011. Ces évènements ont précipité la chute du premier ministre Kankuie Tanaka, plus tard condamné pour corruption.

Ensuite, la diplomatie américaine a déclenché une campagne pour convaincre les Nations Unies et d'autres organismes multilatéraux à lutter contre la corruption transnationale, et aussi contre la légitimation fonctionnaliste de la corruption. C'est dans ce but que les Etats Unis ont réussi à convaincre l'OCDE à créer le Groupe ad hoc sur les paiements illicites (1989), plus tard nommé Groupe de travail sur la corruption de l'OCDE ${ }^{13}$.

Sur le plan politique, les États-Unis invitaient les principaux

13 Juliette Lelieur et Mark Pieth. Dix ans d'application de la convention OCDE contre la corruption transnationale. In Reccueil Dalloz. Paris : 2008, p. 1086. 
concurrents - France, Japon, Allemagne, Royaume-Uni - à souscrire des mesures concrètes contre le versement de pots-de-vin à des agents publics étrangers dans le cadre des transactions transnationales, sous la menace d'abroger le Foreign Corrupt Practices Act. Après des négociations assez difficiles, les Etats membres de l'OCDE, ainsi que six Etats partenaires (y compris le Brésil), ont accepté de coopérer. Sur le plan juridique, l'OCDE a proposé un instrument de soft law : la Recommandation initiale du Conseil sur la corruption dans le cadre de transactions commerciales internationales (1994). L'organisme a publié d'autres textes complémentaires jusqu'à la rédaction d'un texte hard law : la Convention sur la lutte contre la corruption d'agents publics étrangers dans les transactions commerciales internationales du 13 décembre $1997^{14}$.

Le parcours pour écarter le principe de déductibilité fiscale des pots-de-vin dans les transactions commerciales a été long. C'est le résultat de graves préoccupations morales et politiques. Martine Millet-Einbinder observe que la déductibilité est économiquement coûteuse, " parce qu'elle entrave le développement des échanges et des investissements internationaux en augmentant les coûts de transaction et en faussant les conditions de concurrence ${ }^{15}$ ». Dans son article, elle souligne qu'en 1996 la législation de plusieurs pays admettait la déductibilité des pots-de-vin : Allemagne, Australie, Autriche, Belgique, France, Islande, Luxembourg, Nouvelle Zélande, Pays-Bas, Portugal et Suisse, parmi d'autres. Ainsi, l'auteure met en évidence le rôle du Comité des affaires fiscales (CAF), une importante instance de politique fiscale de l'OCDE, qui a commencé ces activités en juin 1994 dans le but de persuader les pays européens à refuser la déductibilité ${ }^{16}$.

Pendant longtemps, la corruption politique s'appuyait sur une certaine tolérance de la société. Les « transactions », assez complexes et secrètes, étaient à l'abri de l'opinion publique, des autorités fiscales et du Ministère Public. Elles n'étaient considérée qu'un « crime blanc », étant donné la faiblesse de la réaction sociale, des sanctions

14 Juliette Lelieur et Mark Pieth. Dix ans d'application de la convention OCDE contre la corruption transnationale. In Reccueil Dalloz. Paris : 2008, p. 1087.

15 Martine Millet-Einbinder. La non-déductibilité des pots-de-vin versés à des agents publics étrangers. In : Affairisme : la fin du système - Comment combattre la corruption. Paris : OCDE, 2000, p. 73.

16 «En pratique, ces pays refusaient souvent la déduction lors des vérifications fiscales, faute de justificatifs suffisants quant à la nécessité de la dépense pour la réalisation du revenu. Plus précisément, la déductibilité des pots-de-vin versés à des agents publics étrangers était généralement subordonnée à la divulgation de l'identité du bénéficiaire aux autorités fiscales lorsque le contribuable est réticent à faire suite à cette demande » Cf. Martine Millet-Einbinder. La non-déductibilité des pots-de-vin versés à des agentes publics étrangers. In : Affairisme : la fin du système - Comment combattre la corruption. Paris : OCDE, 2000, p. 79. 
et du coût social ${ }^{17}$. En plus, il y avait la perception de l'impunité chez les hommes politiques. Cette situation a commencé à changer à partir des grosses affaires judiciaires et financières amplement diffusées dans les médias. Les scandales du monde politique ont provoqué une profonde indignation populaire et ont exposé les fragilités, voire les dysfonctions de l'État et des institutions démocratiques ${ }^{18}$. L'exemple le plus emblématique a été l'opération Mains propres, qui a eu lieu en Italie au début des années 90 : une série d'enquêtes dont le but était de démanteler le système de corruption et le financement illicite des partis politiques, incrusté dans l'administration publique et dans le secteur privé, surtout l'influence des mafias dans le « Tangentopoli ${ }^{19}$ ».

Force est de conclure que la question éthique n'a pas été la principale raison de la lutte contre la corruption transnationale menée par l'OCDE. Au début, c'est l'occupation des marchés publics qui a rassemblé des pays européens afin d'adopter des mesures de prévention et de répression aux dessous-de-table versés à des fonctionnaires étrangers par les entreprises. Les aspects éthiques de ces échanges n'étaient que transversaux dans le discours officiel, conçu davantage pour convaincre l'opinion publique que pour le bien fondé de la réglementation. La moralité administrative est devenue une valeur majeure à partir des années 80 , quand la corruption commence à se mêler à d'autres infractions comme les mafias, le blanchiment d'argent, le financement de partis politiques et de campagnes électorales, dont les scandales et les affaires criminelles ont attiré l'intérêt de la presse et des citoyens.

\section{Approche sociologique}

Le champ de recherches sociologiques sur les effets de la corruption est très vaste. Plusieurs études analysent le phénomène selon des perspectives théoriques particulières. On rappellera les auteurs classiques tels que Robert Kiltgaard, Kemp Ronald Hope et Arnold Heidenheimer. Mais il y en a d'autres qui ont apporté des approches très importants pour la compréhension de ce phénomène. Sans avoir la prétention d'exposer tous les résultats des recherches sur ce sujet, cette présente Recherche propose juste une réflexion sur la sociologie de la corruption politique, afin de montrer l'ampleur du problème.

Le vocable corruption vient du latin corruptione, mot qui évoque l'idée de dégénérescence, de putréfaction, de décomposition, de débauche, de subornation ou de dessous de table. Il est de nos jours

17 Lascoumes, Pierre. Corruptions. Paris : Presses de Sciences Po, 1999, p. 11.

18 Brigitte Henri. Le secteur financier : un partenaire important dans la lutte contre la corruption. In Revue Nouvelles Études Pénales, n. 25, 2014, p. 98.

19 «Ville des pots-de-vin », affaire sur la responsabilité du Parquet de Milan. 
employé pour désigner la conduite d'une autorité qui abuse de sa charge pour obtenir des gains privés ou léser les coffres publics.

La corruption est en quelque sorte une criminalité occulte. Son modus faciendi porte l'empreinte de la clandestinité et du secret. Les transactions illicites exigent des protagonistes une discrétion absolue. Personne ne parle, personne ne voit, personne n'entend, telle est la règle. Corrompu et corrupteur se tiennent à l'écart de toute publicité pour éviter les investigations des audits du Fisc, de la Cour des Comptes et du Ministère Public. Un manteau de silence protège les règlements, aussi illégitimes, aussi risqués soient-ils. C'est pourquoi il est si difficile de connaître la réelle dimension des délits pratiqués au niveau national et international.

Les statistiques présentées se fondent sur des données estimatives qui ne correspondent guère à la réalité, des données extraites, par exemple, des services publics ou recueillies auprès des médias. Il existe un abîme profond entre la criminalité apparente (captée par les organes de contrôle social) et la criminalité réelle (actes concrets de corruption). Ce manque d'informations nous empêche d'avoir une idée exacte de l'échelle de corruption pratiquée au niveau national et international. C'est pourquoi plusieurs auteurs utilisent l'expression " chiffre noir » pour désigner cet abîme entre la réalité sociale et les poursuites pénales en matière de corruption.

Comme l'observe Jean-Pierre Bueb, il n'existe, encore, aujourd'hui, aucune statistique qui permette de mesurer l'ampleur des violations de la réglementation concernant les marchés publics. Mais l'absence de données n'autorise guère à affirmer ni que les dérives sont marginales, ni que tous les marchés sont l'objet de détournements. Cet auteur remarque que « cela signifie simplement que l'on ne dispose pas d'indicateurs fiables permettant de quantifier les malversations ${ }^{20} \gg$.

Par contre, la médiatisation des affaires de corruption a exposé les coulisses du pouvoir au grand public. La conséquence la plus évidente est la « crise de la représentation politique ». Ce phénomène s'explique par la perplexité des citoyens devant les scandales liés aux atteintes à la probité publique comme, par exemple, les financements occultes des partis et des campagnes électorales, et aussi le clientélisme, le trafic d'influence, le népotisme et le favoritisme. Les études sur la défense de la probité dans la politique et dans les services publics remontent au début du XXe siècle aux États-Unis. Mais la réaction de la société civile contre la corruption est beaucoup plus récente. Le concept de morale dans la politique prend force et commence à s'incorporer au jugement des électeurs, de plus en plus informés des abus du pouvoir et de la nocivité des pratiques illicites. La presse libre et investigatrice

20 Jean-Pierre Bueb. La lutte contre la fraude et la corruption dans les marchés publics. Forum Mondial de l'OCDE sur la gouvernance - partager les enseignements de la promotion de la bonne gouvernance et de l'intégrité dans le marchés publics. 2006. 
démasque les arrangements et permet aux citoyens de sanctionner électoralement les élus, en les écartant du pouvoir. Ce raisonnement s'insère dans la "théorie de l'électeur rationnel ", selon laquelle les vertus telles l'intégrité, la moralité, l'honnêteté du candidat et le bon usage de la fonction sont des facteurs déterminants dans le vote. Paradoxalement, les recherches montrent que la sanction électorale est encore faible. Plusieurs personnes accusées d'avoir commis des actes de corruption sont réélues, en dépit de la large diffusion des affaires par la presse. Cette constatation nous invite à réfléchir sur les composantes morales du jugement politique ${ }^{21}$.

Les Constitutions et les traités internationaux renforcent les fonctions du pouvoir judiciaire afin de le doter de tous les instruments nécessaires pour lutter contre la corruption. C'est le cas du Brésil, où la « judiciarisation de la politique » est un phénomène inscrit dans le cadre de la jurisprudence, soit pour garantir les droits sociaux, soit pour punir les agents publics malhonnêtes.

La difficulté, voire l'impossibilité, de mesurer le niveau de corruption dans des pays a inspiré la Transparency International à adopter une méthodologie : l'Indice de perception de la corruption (l'IPC). Il s'agit d'un sondage annuel sur la façon par laquelle les personnes - surtout les experts de ce sujet - perçoivent le niveau de corruption dans différents pays. L'IPC est très prestigieux chez les économistes, sociologues et politologues, qui l'utilisent souvent dans ces travaux de recherches.

Cette organisation non-gouvernementale propose une définition concise : « la corruption est un abus de pouvoir reçu en délégation à des fins privées ». Elle présuppose, d'abord, un acte (action ou omission) considéré comme un abus de pouvoir. Ensuite une pratique en faveur d' intérêts privés, c'est-à-dire, l'agent public, sa famille, ses proches, le parti politique, des campagnes électorales, etc. Finalement, l'utilisation indue du pouvoir reçu en délégation (émané du secteur public ou du secteur privé).

La perception de ce phénomène fait partie d'une méthodologie ayant pour but de présenter le portrait le plus proche possible de la réalité. C'est une tâche assez difficile, car les chercheurs doivent affronter plusieurs difficultés, y compris l'invisibilité des comportements transgressifs et la complicité des acteurs des opérations. Comme il est impossible d'avoir des données exactes, les recherches ne prennent en considération que des points de vue de certaines personnes biens placées pour évaluer le comportement des agents publics quelles

21 Les perceptions et jugements sur les atteintes à la probité publique ont été étudiés par Philippe Bezes et Pierre Lascoumes. Cf. « Percevoir et juger la corruption politique - enjeux et usages des enquêtes sur les représentations des atteintes à la probité publique ». In Revue française de Science Politique, vol. 55, n. 5-6, octobre-décembre 2005, p. 757-786. 
estiment corrompus.

Puisqu'il est encore difficile d'avoir des données objectives pour évaluer l'ampleur de ce phénomène, la Transparency International a développé une méthodologie ancrée sur la perception des experts à l'égard de la corruption. À partir des informations recueillies, il est possible de mesurer le manquement de probité dans plusieurs pays. En fait, la pluralité et la corrélation de différentes sources - autorités, chefs d'entreprises, banques, centres de recherches, universités, etc. assurent une grande fiabilité à l'IPC, ce qui justifie son énorme prestige au niveau international ${ }^{22}$.

\section{E. Approche normative}

Le système supranational de combat contre la corruption s'articule en deux niveaux : universel et régional. A l'échelle universelle, il dispose de deux documents législatifs fondamentaux : la Convention des Nations Unies contre la corruption (Résolution 58/4, de l'Assemblée générale du 31 octobre 2003) et la Convention sur la lutte contre la corruption d'agents publics étrangers dans les transactions commerciales internationales, adoptée par la Conférence de négociations de l'OCDE, le 21 novembre 1997. Au niveau régional, il existe le modèle interaméricain, dont le Brésil fait partie. En 1996, les États membres de l'OEA ont souscrit la Convention interaméricaine contre la corruption.

Il s'agit d'une réponse du droit international public à l'accroissement vertigineux de la criminalité économique dans presque tous les pays du monde. Les conventions mentionnées ont pour but de sanctionner les atteintes à l'ordre financier, économique et social, surtout la pratique d'infractions comme la corruption, le blanchiment de capitaux, les fraudes aux subventions publiques, la contrefaçon, le trafic d'influence, l'escroquerie, l'enrichissement illicite et d'autres formes de manque de probité des fonctionnaires publics.

Il s'agit là d'infractions qui présentent une évidente dimension internationale, dont la prévention et la répression exigent l'effective coopération entre les Etats, la mutualisation de moyens d'enquête et de persécution pénale. Elles s'insèrent dans l'espèce que l'on appelle borderless crime (délits sans frontières), parce que les criminels utilisent les grands circuits internationaux pour cacher et nettoyer l'argent sale, produit de la corruption et du crime organisé.

Le concept traditionnel et étroit de corruption active, selon lequel l'autorité corrompue est celle qui accepte ou sollicite une somme d'argent ou tout autre avantage pour favoriser un particulier, personne physique

22 Transparency International. Rapport mondial sur la corruption 2005. Corruption dans le secteur de construction et de la reconstruction d'après-guerre. Paris : Economica, 2005, p. 297. 
ou morale ne sera pas retenu. Pas même celui de corruption passive, celle où le particulier offre au fonctionnaire un pot-de-vin pour obtenir des " faveurs ", faciliter les choses ou contourner certains obstacles bureaucratiques. Certes, il y aura toujours un marché illicite et punissable entre agent public et particulier. Mais il ne faut pas oublier qu'aujourd'hui la corruption acquiert des formes sophistiquées, diverses et complexes, dont la mise en ouvre est engendrée avec beaucoup de créativité.

En matière de criminalité financière il est très important de distinguer la petite de la grande corruption. La première implique des sommes modestes, de petits fonctionnaires et des intérêts locaux. Elle se manifeste dans des relations quotidiennes entre services publics et usagers sous forme de « cadeaux », " gratifications » ou une autre forme d'avantage indue en échange d'un service public gratuit, y compris la pratique d'extorsion. La Transparency International souligne quelques circonstances favorables à l'épanouissement de cette espèce de corruption, parmi lesquelles on peut citer l'informalisation des services publics, la lenteur des procédures, les files d'attente, la manipulation des normes et des règlements, la déshumanisation et privatisation de l'administration ${ }^{23}$.

Les conventions internationales n'ont pas vocation à combattre la petite corruption (administrative), qui se déroule quotidiennement entre fonctionnaires corrompus et usagers, et dont les sommes en jeu sont modestes, malgré sa néfaste nocivité sociale et éthique. Le droit interne dispose de plusieurs mécanismes juridiques pour combattre ce genre de criminalité : le droit pénal, civil et administratif, par exemple.

C'est la grande corruption qui est la principale cible de la législation supranationale. Selon la Banque Mondiale, il s'agit d'infraction « à haut niveau, celui où les décideurs politiques créant et appliquant les lois utilisent leurs positions officielles pour promouvoir leur bien-être, leur statut ou leur pouvoir personnel ». Elle implique des entreprises multinationales responsables pour le versement de sommes faramineuses à des agents gouvernementaux et à des hommes politiques qui sont au sommet de l'Etat pour obtenir des contrats commerciaux lucratifs dans des marchés publics. Les profits alimentent les classes dirigeantes, avides de se perpétuer au pouvoir politique et économique. Elle devient institutionnelle ou systémique lorsque l'État manque de moyens pour les combattre, par le truchement surtout du contrôle judiciaire et des instances autonomes, du Ministère Public indépendant, de la presse libre et de la société civile vigilante.

La corruption n'est pas une infraction simple à définir. Le concept traditionnel chez les auteurs classiques du droit pénal est insuffisant pour expliquer ce phénomène si complexe de la contemporanéité. On parle d'un crime à facettes auquel s'ajoutent d'autres infractions pénales

23 Transparency international. Combattre la corruption - Enjeux et perspectives. Paris : Karthala, 2000, p. 21-24. 
jumelles. Jacques Borricand évoque quelques formes de corruption. La corruption individuelle, destinée à des fins personnelles, tel que l'enrichissement illicite. Elle est occasionnelle et a des objectifs privés. Le fonctionnaire profite de son espace de pouvoir discrétionnaire pour demander ou recevoir des pots-de-vin. La corruption en réseaux est beaucoup plus complexe, parce qu'elle exige un niveau élevé d'organisation. Elle met en mouvement des sommes pharaoniques, produit de multiples infractions complémentaires. En général, un secteur est élu pour des actions criminelles : ministères, mairies, entreprises publiques, appels d'offres de biens et de services etc ${ }^{24}$.

Selon Jacques Borricand, il existe deux espèces de corruption en réseaux : la corruption organisée et la corruption-chantage. La première implique des agents publics et des entreprises qui s'articulent pour obtenir des avantages illicites en utilisant des facilités, des privilèges ou des trucages dans des procédures administratives. Dans ce modèle, il est très difficile distinguer le corrupteur du corrompu, puisqu'ils agissent en absolue complicité. La deuxième espèce a comme facteur déterminant le but d'obtenir des fonds pour soutenir des structures de pouvoir : financement de partis politiques et de campagnes électorales à l'échelle nationale ou régionale. L'engagement partisan, la solidarité, la réciprocité et l'ambition de prendre ou de maintenir le pouvoir politique est à l'origine de cette forme de criminalité. Les sociétés qui détiennent des portions de marchés publics sont obligées vis-à-vis des représentants des partis politiques pour éviter des représailles ${ }^{25}$. Face à la complexité de la criminalité financière, il est parfois difficile de les distinguer avec précision.

Le mot corruption sera donc employé dans un sens plus large (la grande corruption), qui consiste en un ensemble d'infractions pénales : la criminalité économique, le transfert de fonds d'origine illicite provenant d'infractions contre l'Administration publique, le blanchiment d'argent, le financement illicite des partis politiques et des campagnes électorales, le détournement de fonds et de biens appartenant au patrimoine public, le trafic d'influence, le favoritisme, « les dessous-de- table », l'abus de fonctions, l'extorsion, l'enrichissement illicite des agents publics et des hommes politiques.

Les États ont le devoir de signer et de ratifier les conventions contre la corruption. Elles leurs imposent des prestations positives contre cette forme de criminalité, tout en respectant la souveraineté de chaque pays. Ils doivent également adopter les mesures législatives, administratives et judiciaires qu'ils jugent nécessaires pour honorer

24 Jacques Borricand. Corruption et Politique. In Problèmes Actuels de Science Criminelle, n. IX. Aix-En-Provence : Presses Universitaires d'Aix-Marseille, 1996, p. 67.

25 Jacques Borricand. Corruption et Politique. In Problèmes Actuels de Science Criminelle, n. IX. Aix-En-Provence : Presses Universitaires d'Aix-Marseille, 1996, pp. 72-73. 
les principes supranationaux établis, d'une manière compatible avec l'égalité souveraine, l'intégrité territoriale, la non-intervention et l'autodétermination des peuples. Ce devoir d'harmonisation législative et d'application rigoureuse des lois anticorruption sont des démonstrations inéquivoques de l'attachement du pays aux engagements assumés sur le plan international.

Il est vrai que la corruption est devenue un crime de dimension transfrontière, qui demande la coopération internationale. Mais il est également vrai que la pratique de l'infraction se développe à cause de la faiblesse du contrôle interne de la gestion administrative sur les dépenses gouvernementales, favorisant l'enrichissement illicite des agents publics, le financement des partis politiques et les trucages dans l'attribution de marchés publics et le détournement de fonds ${ }^{26}$. L'intervention de l'ordre juridique supranational est nécessaire pour harmoniser les législations internes et pour donner plus d'efficacité à la poursuite pénale.

\section{LE SYSTÈME INTERAMÉRICAIN E LA LUTTE CONTRE LA CORRUPTION}

Le Brésil est l'un des 35 pays membres de l'Organisation des États Américains (OEA $)^{27}$, l'institution régionale la plus ancienne au monde. L'idée de sa création remonte à la fin du XIX siècle, lors de la Conférence internationale américaine, qui s'est déroulée à Washington (1889-1890). Mais elle a commencé à exister en tant qu' organisation supranationale en 1948, quand la Charte de l'OEA a été signée. Au fil des années elle a été perfectionnée par plusieurs amendements. L'ensemble des traités internationaux et des dispositions normatives est nommé « Système interaméricain ${ }^{28}$ ».

Ayant son siège à Washington, l'OEA accueille la Commission Interaméricaine de Droits de l'Homme, l'Assemblée générale, le Comité juridique interaméricain, ainsi que d'autres entités et organismes spécialisés. En tant qu'institution régionale, elle a pour but d'assurer à tous les Etats membres « un ordre de paix et de justice, de maintenir

26 M. Carlos CASTRESANA FERNANDEZ. Rapport Général. La corruption dans les marchés publics. Allemagne : Edition du Conseil de l'Europe, 1998, p. 29.

27 Du point de vue territorial, l'OEA rassemble des pays de l'Amérique du Sud, de l'Amérique du Nord, de l'Amérique Centrale et des Caraïbes. Les États qui ont signé la Charte de l'OEA: l'Argentine, la Bolivie, le Brésil, le Chili, la Colombie, le Costa Rica, Cuba, la République Dominicaine, l'Equateur, El Salvador, Le Guatemala, Haïti, le Honduras, le Mexique, le Nicaragua, le Panama, le Paraguay, le Pérou, Les États-Unis de l'Amérique, l'Uruguay et le Venezuela. Ultérieurement, d'autres pays l'ont adhéré entre 1967 et 1991 : Barbade, Trinité-etTobago, Jamaïque, Grenade, Suriname, Dominique, Sainte Lucie, Antigua et Barbuda, SaintVincent-et-Grenadines, Bahamas, Saint-Kitts-et-Nevis, Canada, Belize et Guyana.

28 Sur la creation de l'OEA : www.oas.org. 
leur solidarité, de renforcer leur collaboration et de défendre leur souveraineté, leur intégrité territoriale et leur indépendance (article 1)».

Ces caractéristiques font de l'OEA le principal forum multilatéral de protection des droits de l'homme, des valeurs démocratiques, et aussi du combat contre le terrorisme, la pauvreté, les inégalités sociales, la corruption endémique au niveau régional, et pour la diversité culturelle.

D'autres systèmes régionaux ont aussi adopté des conventions contre la corruption. Il suffit de les mentionner, puisque ils ne sont pas l'objet de cette recherche. Le Conseil de l'Union Européenne a adopté la « convention relative à la lutte contre la corruption impliquant les fonctionnaires des Etats membres de l'Union Européenne (1997). Ensuite, la « Convention pénale sur la corruption du Conseil de l'Europe (1999)» et la « Convention civile sur la corruption du Conseil de l'Europe ». En Afrique, l'Organisation de l'unité africaine OUA - composée de 53 Etats - a souscrit à la «Convention de l'Union Africaine sur la prévention et la lutte contre la corruption (2003)». Enfin, simple rappel, la Ligue des États Arabes a adopté au Caire, le 21 décembre 2010, la « Convention arabe contre la corruption », ratifiée par plusieurs pays.

Les systèmes régionaux et la lutte contre la corruption :

\begin{tabular}{|l|l|l|l|l|}
\hline Système & Organisme & \multicolumn{1}{c|}{ Convention } & Signature & \multicolumn{1}{|c|}{ Vigueur } \\
\hline Européen & $\begin{array}{l}\text { Convention relative à } \\
\text { la lutte contre la } \\
\text { corruption impliquant } \\
\text { les fonctionnaires des } \\
\text { Etats membres de } \\
\text { l'Union Européenne } \\
\text { Convention pénale } \\
\text { l'Europe } \\
\text { sur la corruption; } \\
\text { Protocole additionnel à } \\
\text { la Convention pénale; } \\
\text { Convention civile } \\
\text { sur la corruption; }\end{array}$ & 1999 & 2003 & 2005 \\
\hline $\begin{array}{l}\text { Interamé- } \\
\text { ricain }\end{array}$ & $\begin{array}{l}\text { Organisation } \\
\text { des Etats } \\
\text { américains }\end{array}$ & $\begin{array}{l}\text { Convention } \\
\text { interaméricaine } \\
\text { contre la corruption }\end{array}$ & 1999 & 2005 \\
\hline Africain & $\begin{array}{l}\text { Organisation } \\
\text { de l'unité } \\
\text { africaine }\end{array}$ & $\begin{array}{l}\text { convention de l'Union } \\
\text { Africaine sur la } \\
\text { prévention et la lutte } \\
\text { contre la corruption }\end{array}$ & 2003 & 1997 \\
\hline Arabe & $\begin{array}{l}\text { Ligue des } \\
\text { Etats Arabes }\end{array}$ & $\begin{array}{l}\text { Convention arabe } \\
\text { contre la corruption }\end{array}$ & 2010 & $\begin{array}{l}\text { Après } \\
\text { ratification } \\
\text { et dépôt de } \\
\text { l'instrument de } \\
\text { chaque État. }\end{array}$ \\
\hline
\end{tabular}




\subsection{La Convention Interaméricaine contre la corruption}

En 29 mars 1996, l'OEA a adopté la Convention interaméricaine contre la corruption (en vigueur à partir du 6 mars 1997). Ce document est à l'origine d'un système intégré de prévention et de répression de la grande corruption. D'autres conventions ont été édictées sur le plan régional : Convention interaméricaine sur l'entraide judiciaire en matière pénale (1992) et protocole facultatif additionnel (1993) ; Convention interaméricaine sur l'exécution de condamnations pénales à l'étranger (1993) ; Convention interaméricaine sur commissions rogatoires (1975) et son protocole facultatif additionnel (1979).

Le Brésil et le système interaméricain contre la corruption :

\begin{tabular}{|c|c|c|c|c|}
\hline Organisme & Convention & Signature & Ratification & Décret \\
\hline OEA & $\begin{array}{l}\text { Convention Interaméricaine } \\
\text { contre la corruption }\end{array}$ & 29/03/1996 & 07/10/2002 & 4.410 \\
\hline OEA & $\begin{array}{l}\text { Convention interaméricaine } \\
\text { sur l'entraide judiciaire } \\
\text { en matière pénale }\end{array}$ & 23/05/1992 & 03/01/2008 & 3.340 \\
\hline OEA & $\begin{array}{l}\text { Protocole additionnel à la } \\
\text { Convention interaméricaine } \\
\text { sur l'entraide judiciaire } \\
\text { en matière pénale }\end{array}$ & $23 / 05 / 1992$ & $03 / 01 / 2008$ & 3.340 \\
\hline OEA & $\begin{array}{l}\text { Convention interaméricaine } \\
\text { sur l'exécution de } \\
\text { condamnations } \\
\text { pénales à l'étranger }\end{array}$ & 09/06/1993 & $12 / 07 / 2006$ & 5.519 \\
\hline OEA & $\begin{array}{l}\text { Convention interaméricaine } \\
\text { sur des commissions } \\
\text { rogatoires }\end{array}$ & $30 / 01 / 1975$ & 09/05/1996 & 1898 \\
\hline OEA & $\begin{array}{l}\text { Protocole additionnel à la } \\
\text { Convention interaméricaine } \\
\text { sur commissions rogatoires }\end{array}$ & 08/05/1979 & 07/10/1996 & 2.022 \\
\hline
\end{tabular}

La Convention interaméricaine contre la corruption, adoptée par les États membres de l'OEA, est la première réponse régionale à la criminalité financière, très présente dans des pays de l'Amérique du Sud, de l'Amérique Centrale et des Caraïbes. Les États-Unis et le Canada ont aussi connu de grosses affaires de corruption, qui ont beaucoup bouleversé leur stabilité économique et politique.

Les pays membres sont persuadés que la corruption dégrade la légitimité des institutions politiques, porte atteinte au développement des peuples, augmente les inégalités sociales, empêche la mise-enœuvre des politiques publiques efficientes et de bonne qualité, renforce 
les actions des organisations criminelles et menace des régimes démocratiques. Le combat contre la corruption transnationale s'impose comme l'une des stratégies les plus importantes pour assurer l'entraide entre les États, dans le but d'identifier et de réprimer les infractions qui sont à l'origine du problème. En résumé, La Convention encourage les pays à renforcer les mécanismes nécessaires pour prévenir, dépister, sanctionner et éliminer la corruption.

D'abord, la Convention Interaméricaine propose quelques définitions et précisions sur des mots et des expressions employés dans son texte, notamment sur le contenu du crime de corruption national et transnationale. Ensuite elle présente quelques mesures de prévention comme, par exemple :

- l'adoption de normes déontologiques de conduite pour l'exercice de la fonction publique, parmi lesquelles le devoir de rapporter aux autorités les actes de corruption dont quelque fonctionnaire aurait connaissance ;

- le devoir de déclarer les revenus, avoirs et dettes lors de l'exercice d'une fonction dans l'administration publique ;

- la protection des fonctionnaires qui dénoncent des actes de corruption, y compris la protection de leur identité ;

- la participation de la société civile aux efforts pour la combattre.

Sur le plan pédagogique, elle incite les pays à prendre des mesures visant à dissuader la pratique de corruption des fonctionnaires nationaux ou étrangers, à partir de systèmes de contrôle comptables, $\mathrm{y}$ compris des registres et détails des transactions financières concernées.

Elle invite les pays membres à sanctionner l'enrichissement illicite des fonctionnaires publics. Le droit interne doit conférer le caractère d'infraction à l'augmentation significative du patrimoine d'un agent public, qui n'a pas les moyens d'expliquer raisonnablement les biens acquis pendant l'exercice de ses fonctions. Au Brésil, par exemple, la Loi 8.429/92 a un caractère civil, mais impose des peines sévères comme la perte de la fonction publique, la suspension des droits politiques, la réparation du préjudice, les dommages moraux et l'interdiction de signer des contrats avec l'État ou avec des entreprises publiques. En vertu de la rigueur de ses sanctions, plusieurs juristes brésiliens la considèrent comme une loi formellement pénale (penaliforme), thèse rejetée par le Tribunal Fédéral Suprême (STF).

La Convention a élu le "principe de la progressivité ", autrement dit, les pays membres sont tenus d'adopter dans un délai raisonnable l'harmonisation des législations nationales afin de lutter plus efficacement contre la corruption, à partir de l'adoption de réponses pénales, civiles et administratives. Sans doute, les pays membres de l'OEA ont-ils fait de larges progrès dans ce domaine, malgré les grosses 
affaires de corruption diffusées par la presse. Aujourd'hui, le problème n'est pas dans le manque de lois, mais dans leur faible effectivité.

L'extradition des étrangers est un autre aspect qui a mérité l'attention de l'OEA. D'abord, l'organisme stimule les pays à signer des traités internationaux concernant l'extradition, dans lesquels les infractions sont prévues. Mais en l'absence de traité, les parties peuvent utiliser la Convention comme base légale d'extradition (article XIII 3).

L'entraide et la coopération est un autre sujet d'importance. Les pays s'accordent l'assistance mutuelle la plus étendue, et aussi la coopération technique au sujet de procédures et de méthodes considérées les plus efficaces pour la prévention, le dépistage et la sanction des actes de corruption (article XIV). En ce qui concerne la protection de la vie privée, les Etats ne peuvent pas refuser la collaboration sollicitée sous prétexte de protection du secret bancaire.

\subsection{La Convention interaméricaine sur l'entraide judiciaire en matière pénale}

Cette convention a pour but de renforcer le régime d'entraide entre les Etats parties de 1'OEA. On peut remarquer, d'abord, qu'elle incite les pays à adopter le système de double incrimination. Mais l'assistance est accordée même si le pays requis ne considère pas comme infraction l'acte commis à l'étranger. Pourtant, il ne peut pas prendre de mesures coercitives - saisie, séquestre de biens, perquisition et confiscation de biens, par exemple - si le crime n'est pas prévu dans sa législation. Voilà la raison pour laquelle l'harmonisation progressive des régimes juridiques est essentielle à l'efficacité du modèle interaméricain.

L'article 7 de la Convention présente les formes les plus courantes d'entraide entre les Etats. Elles se développent au niveau judiciaire à partir de l'exécution d'actes de procédures, in verbis :

- a. Notification des décisions judiciaires et des jugements ;

- b. Réception de témoignages et de déclarations de personnes;

- c. Citations de témoins et d'experts aux fins de déposition;

- d. Exécution de saisies et de séquestre de biens, immobilisation d'actifs et assistance aux procédures relatives à la confiscation ;

- e. Réalisation d'inspections et de saisies ;

- $\mathrm{f}$. Présentation de pièces judiciaires ;

- g. Remise de documents, de rapports, d'informations et des preuves ;

- h. Transfèrement des personnes détenues, aux effets de la présente Convention ;

- i. Tout autre acte de procédure pourvu qu'un accord soit intervenu entre l'État requérant et l'Etat requis. 
Toutes ces mesures doivent respecter le principe de la réciprocité, qui est l'âme de l'entraide judiciaire internationale. Il est parfaitement légitime de refuser de communiquer des renseignements si l'État requérant méprise tel principe, en empêchant des poursuites dans des circonstances similaires. Evidemment, toutes les Constitutions contemporaines reconnaissent et protègent les libertés fondamentales, parmi lesquelles le droit au procès équitable, l'égalité des armes, le contradictoire et surtout le droit de ne pas témoigner contre soi-même. En général, ce ne sont pas des prérogatives absolues. Parfois, elles sont limitées devant l'importance des affaires de corruption, puisque l'argent détourné va certainement dégrader l'économie nationale et porter atteinte aux politiques publiques dans le domaine de la santé, de l'éducation, du logement, du transport collectif, de l'infrastructure etc. De plus les informations sur des personnes morales n'ont pas la rigidité des garanties attribuées aux personnes physiques.

La Convention interaméricaine sur l'entraide est très spécifique en ce qui concerne les aspects procéduraux. La notification de décisions, d'arrêts, de jugements, ainsi que la comparution de témoins et d'experts, la remise d'informations et d'antécédents occupent une place privilégiée dans le texte. Elle prévoit aussi le transfèrement d'une personne objet d'une procédure pénale, à la condition qu'elle donne son consentement, et que les États respectent d'autres conditions prévues dans les articles 21 et 22. Il existe, dans l'article 9 , des hypothèses où les pays peuvent refuser l'entraide judiciaire. Ce sont des cas qui impliquent les délits politiques, la demande d'un tribunal d'exception ou d'un tribunal ad hoc, des demandes envisageant des actions discriminatoires contre des groupes en raison de leur sexe, race, condition sociale, nationalité, religion et appartenance idéologique.

\section{PolitiQue CRiminelle internationale ANTi-CORRUPTION}

Les secrets du pouvoir. Voilà un sujet qui a inspiré plusieurs ouvrages, qui attire l'intérêt des médias et qui est au cœur des débats académiques. À partir des années 90, les coulisses du monde politique ont été dévoilées, exposant toutes les fractures. Soudain, les citoyens se sont rendus compte que les élus méprisaient les valeurs républicaines. Les affaires publiques sont devenues une source d'enrichissement illicite et de financement de campagnes électorales. Plusieurs scandales financiers ont bouleversé la conception archétypique de la démocratie en tant que régime représentatif du contrat social, dont la volonté générale et le bien commun sont ses principaux fondements. Eva Joly rappelle que « les enquêtes judiciaires en Europe, que ce soit en Italie, en Espagne ou en Allemagne, démontrent des fonctionnements collectifs défaillants et des mécanismes criminels au cœur du pouvoir. 
Nous pensions, jusqu'à présent, que le crime vivait dans l'ombre de nos sociétés. Nous le trouvons intimement lié aux grandes entreprises ou aux dirigeants politiques européens les plus honorables ${ }^{29}$ ». Cette constatation a changé pour toujours la perception populaire de la notion de criminalité. Ainsi la sacralisation du pouvoir a donné lieu à une insurmontable méfiance de l'opinion publique envers les élus et, dans certains cas, au phénomène de la criminalisation de la politique.

S'il est vrai que le système mondial de lutte contre la corruption s'est largement développé depuis 1997, il est aussi vrai que la faible effectivité des cadres normatifs nationaux et internationaux dans certains pays, y compris le Brésil, encourage le versement de potsde-vin en échange de marchés publics. Malgré la vaste diffusion des scandales par des médias, les pratiques corrompues augmentent d'une façon préoccupante dans la majorité des pays développés et en développement, exigeant des réponses efficaces sur le plan juridique et gouvernemental, sans oublier d'autres partenaires comme des organisations multilatérales, le secteur privé et la société civile.

A partir de la Convention de l'OCDE, la communauté internationale commence à s'articuler autour des documents normatifs et des actions concrètes pour lutter contre la corruption. Parmi les mesures adoptées, il convient de citer l'entraide judiciaire à plusieurs niveaux, la responsabilisation pénale et civile des personnes morales, la répression au blanchiment de capitaux, le renforcement des sanctions contre les agents publics impliqués, la politique de prévention, la pression exercée sur des paradis fiscaux et l'élargissement de la coopération transfrontalière. Les pays signataires des conventions sont obligés d'adapter leurs législations aux nouvelles exigences et de s'engager à les mettre en application.

Plusieurs enquêtes montrent que la corruption dans la passation des marchés publics demeure un problème irrésolu et alarmant. Le versement de pots-de-vin à des fonctionnaires pour l'obtention des marchés publics reste encore une pratique adoptée par des entreprises multinationales, malgré les efforts des organismes multilatéraux, voire de la société civile.

L'opacité des transactions commerciales lors de la passation de marchés publics reste encore la règle. Le devoir de transparence et les dispositions anti-corruption sont toujours contournés par les personnes intéressées, soit des agents publics, soit des entreprises qui cachent leurs véritables intentions avec beaucoup de créativité. Le transfert des sommes versées à des fonctionnaires corrompus est encore possible grâce aux complexes réseaux criminels et à la complicité des paradis fiscaux et judiciaires.

Le cadre normatif supranational est, sans doute, satisfaisant pour

29 Eva Joly. Notre affaire à tous. Paris : Les arènes, 2000, p. 19-20. 
mener un combat efficace contre la corruption. Mais la composante la plus importante est la véritable détermination des détenteurs du pouvoir à vaincre les obstacles que rencontre ce combat: la bureaucratie paralysante, les procédures complexes et longues, les moyens insuffisants pour faire marcher les enquêtes, le corporatisme, les manipulations politiques, parmi d'autres $^{30}$. En conséquence, le vrai problème repose sur la faiblesse de l'effectivité des lois. C'est plutôt une question d'application que de construction normative. Le discours juridique rationnel et post-positiviste doit renforcer l'engagement à assurer le respect à la probité et aux valeurs républicaines dans les affaires publiques.

En effet, la lutte contre la corruption exige l'articulation des actions menées par les gouvernements, les entreprises, les établissements financiers, le pouvoir judiciaire et le ministère public de chaque État. Les organismes de contrôle jouent aussi un rôle très important dans la lutte contre la corruption. D'autres acteurs peuvent aider en tant que sentinelles des entreprises comme, par exemple, les avocats, les comptables, les analystes et les auditeurs.

La lutte contre la corruption a été renforcée par des conventions de l'organisation des Nations Unies et de l'OCDE. Ces organismes invitent tous les pays signataires à harmoniser leurs dispositifs aux nouvelles exigences. Le défi de la p2olitique criminelle dans ce domaine consiste à persuader les États à prendre des mesures législatives concrètes et urgentes pour affronter la délinquance financière de la façon la plus efficace possible. Au plan normatif, l'action politique proposée par la communauté internationale porte sur quatre aspects : 1) l'incrimination ; 2) la prévention ; 3) la détection ; les poursuites et 4) les sanctions.

Politique criminelle internationale anti-corruption

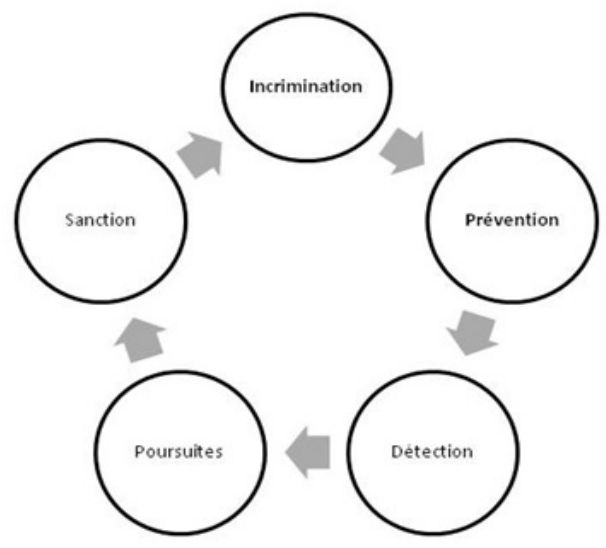

30 Jean-Louis Rocca. La corruption. Paris : Syros, 1993, 128. 
Parmi d'autres initiatives concernant la coopération internationale, l'entraide judiciaire internationale est plus complexe et problématique. Elle est la condition sine qua non pour donner plus d'efficacité à la lutte contre la corruption. Plusieurs conventions et documents consultatifs préconisent des stratégies, des outils et des orientations envisageant d'aider les juges à promouvoir les mesures nécessaires pour affronter ce défi. Le champ de l'entraide judiciaire est extrêmement vaste : collecte des éléments de preuve, application des mesures coercitives demandées par le juge étranger, échanges entre cellules de renseignements financiers, etc. Le refus ou la lenteur des autorités judiciaires dans la mise-en-œuvre des mesures est un facteur qui explique l'impunité, voire l'accroissement de la délinquance financière et de la corruption.

À partir de l'analyse des sources normatives disponibles, il est pertinent de retenir trois niveaux d'intervention judiciaire à l'étranger : 1) mesures provisoires ; 2) mesures procédurales et 3) mesures définitives.

\begin{tabular}{|c|c|c|}
\hline Mesures Provisoires & Mesures Procédurales & Mesures Définitives \\
\hline $\begin{array}{l}\text {-Identification du } \\
\text { produit du crime } \\
\text {-localisation d'avoirs } \\
\text {-gel de capitaux } \\
\text {-saisie des biens } \\
\text {-arrestation de la } \\
\text { personne soupçonée } \\
\text {-rétention du } \\
\text { passeport }\end{array}$ & $\begin{array}{l}\text {-témoignages/ } \\
\text { dépositions } \\
\text {-comparution } \\
\text {-volontaire } \\
\text {-perquisitions } \\
\text {-examiner des objets } \\
\text {-visiter les lieux } \\
\text { envoi de pièces } \\
\text {-à conviction } \\
\text {-estimations } \\
\text { d'experts } \\
\text {-envoi des documents } \\
\text {-cartes rogatoires } \\
\text {-autres mesures. }\end{array}$ & $\begin{array}{l}\text {-confiscation d'avoirs } \\
\text {-restitution des biens } \\
\text { transfèrement des } \\
\text {-personnes détenues } \\
\text {-extradiction } \\
\text { des accusés } \\
\text {-exécution de } \\
\text { condamnations } \\
\text { pénales dans } \\
\text { l'étranger. }\end{array}$ \\
\hline
\end{tabular}

La célérité est l'un des principes-clés de l'entraide judiciaire. Le juge doit prendre les mesures demandées d'une façon rapide, dans les délais les plus raisonnables. L'autorité judiciaire ne peut pas refuser l'entraide sous le prétexte de respecter le secret bancaire. Même si la « double incrimination » reste la règle générale, cette condition n'est pas absolue, puisqu'elle sera considérée remplie sitôt que l'infraction commise dans le pays requérant tombera sous la définition de corruption dans l'État où la mesure judiciaire aura lieu. Mais la conformité et l'harmonisation des lois nationales aux définitions proposées par des 
conventions internationales s'imposent en tant que politique criminelle anticorruption.

\section{Conclusion}

Les formes pour sanctionner la corruption dans les pays signataires des conventions internationales sonttrès variées. En général, les lois prévoient des peines comme les amendes civiles et pénales, l'emprisonnement avec ou sans sursis, la réparation, la confiscation, l'inéligibilité, l'exclusion, la dissolution des entreprises, le programme de conformité, l'exclusion des offres d'appel et la perte de la fonction publique.

Dans le secteur privé, la mise en œuvre des codes professionnels déontologiques est l'un des moyens les plus efficaces pour augmenter la transparence, la conformité et l'intégrité des entreprises. Il s'agit d'un document interne dont le but est de guider les cadres et les employés vers les bonnes pratiques de gouvernance, surtout celle qui assure la réputation positive de la société en vue des transactions transnationales et internes. Dans ce but, elles doivent refuser les pratiques nuisibles comme la formation de cartels et le trucage dans des appels d'offre publics $^{31}$. Les entreprises sont invitées à respecter la législation anticorruption, ainsi que les principes de la bonne gouvernance et la loyauté dans les transactions commerciales, sous risque de subir de sévères sanctions.

Il faut également mettre en valeur le rôle des médias dans la lutte contre la corruption des agents publics. Il est nécessaire d'assurer la liberté d'expression et la liberté de presse dans le sens le plus large possible. La médiatisation des scandales financiers et politiques contribue fermement à la détection, prévention et répression de cette forme de criminalité. Les blogs indépendants sont également une voie très efficace de diffusion des affaires auprès de l'opinion publique. Tous ces moyens sont essentiels à la prise de conscience des citoyens aux dangers de la corruption à l'échelle nationale et internationale.

L'élimination, voire la diminution substantielle, de la corruption exige aussi un fort activisme politique. La société civile joue un rôle essentiel dans ce combat, surtout les organisations nongouvernementales chargées de dénoncer les pratiques corrompues et d'exiger des réponses judiciaires aux abus de pouvoir. Il faut persuader les citoyens que le manque de probité dans l'exercice de la fonction publique est à l'origine de la faible effectivité des droits sociaux dans des pays en développement. Plusieurs secteurs sont affectés comme, par exemple, la santé, l'environnement, l'éducation, les transports, l'habitation, le travail et la qualité de vie de la population.

31 Cf. Transparency International. Rapport mondial sur la corruption 2009. La corruption et le secteur privé. Cambridge : Cambridge University Press, 2009. 
De nos jours, les scandales politiques ont toujours des liens avec la corruption et le financement occulte des campagnes électorales. Cette pratique, incrustée dans la vie institutionnelle de plusieurs pays, est profondément nuisible à la démocratie. Elle porte préjudice à l'économie, sape la confiance des citoyens envers le gouvernement et entrave le développement. L'action politique devient, elle aussi, la cible des suspicions. Les électeurs estiment que les donateurs auront un traitement privilégié dans des affaires publiques, voire à l'occasion de la réglementation des marchés ${ }^{32}$. L'un des outils les plus importants pour assurer la transparence est la divulgation d'informations pour contrôler les flux financiers en politique. Gene Ward souligne qu'elle a pour but d'assurer la comptabilité et de rendre compte des contributions et des dépenses. La diffusion des données permet l'accès à l'information et assure l'égalité d'opportunités dans le processus électoral. Cette procédure permet aux électeurs de qualifier les actes des candidats, voire des partis politiques, en tant qu'activité transgressive portant atteinte à des valeurs et au contrat social.

$\mathrm{Au}$ Brésil, la corruption est devenue un problème politique de grande ampleur, surtout après les affaires « Mensalão » e « Lava Jato ». Des travaux de recherche montrent que l'alliance entre les BTP et le pouvoir politique a commencée pendant le gouvernement Juscelino Kubitschek (1965-1960), lors de la construction de la ville de Brasilia, la nouvelle capitale brésilienne. Certaines entreprises ont renforcé les rapports avec des gouvernements qui se sont succédé. Résultat : elles ont obtenu des contrats faramineux dans les secteurs économiques les plus rentables des marchés publics. Même après la démocratisation du pays, les arrangements ont continué des plus en plus importants. Des entreprises, telles que Mendes Júnior et Odebrecht, parmi d'autres, ont quand même réussi à de gros appels d'offre à l'étranger. Quelques-unes sont devenues des multinationales très puissantes ${ }^{33}$.

Le système de financement privé des campagnes électorales, adopté par le Brésil depuis 1945, a renforcé le prestige des grandes entreprises auprès du pouvoir. Pour obtenir les contrats le plus avantageux, elles versent des millions d'euros pour soutenir des partis politiques susceptibles d'occuper le gouvernement. Cette pratique est incrustée dans la vie institutionnelle du pays et alimente un réseau de corruption qui implique des élus, des ministres, des cadres et des fonctionnaires du haut rang ${ }^{34}$.

32 Gene Ward. Le rôle de la divulgation d'informations dans la lutte contre la corruption dans le financement politique. In : Transparency International. Rapport mondial sur la corruption 2004. La corruption politique : Cambridge University Press, 2004, p.47.

33 Carlos Madeiro. Casamento de empreiteiras com poder começou com JK e teve sua lua de mel na ditadura. www.noticias.uol.com.br. 21/01/2014.

34 Carlos Madeiro. Casamento de empreiteiras com poder começou com JK e teve sua lua de 
A partir des années 80, plusieurs affaires se sont succédé. La plupart implique des hommes politiques et des fonctionnaires de haut rang. La plus importante (Opération Lava Jato) concerne aux malversations à la PETROBRAS, l'entreprise pétrolière contrôlée par l'Etat. Grâce à l'opération menée par le Ministère Public et par la Police Fédérale à mi-novembre 2014, plus connue comme " Jugement dernier », plusieurs PDGs des entreprises du bâtiment et de travaux publiques (BTP), ainsi que des cadres de l'entreprise publique, ont été incarcérés. L'accusation : formation de cartel et corruption. Les sept entreprises soupçonnées ${ }^{35}$ jouent ont rôle très important dans le financement de campagnes électorales et restent en relation avec plusieurs dirigeants de partis politiques. Pour contrôler les marchés publics, les entreprises versaient des commissions de $1 \%$ à $3 \%$ destinées aux partis de la coalition gouvernementale, surtout le Partis des travailleurs (PT), le Parti du mouvement démocratique brésilien (PMDB) et Parti progressiste (PP). Selon le reportage publié dans le journal Le Monde, les entreprises auraient détourné 3 milliards d'euros en dix ans ${ }^{36}$. Seulement pour la campagne électorale de 2014, les entreprises ont versé officiellement 151 millions d'euros, sans parler de l'argent occulte ${ }^{37}$.

Voilà le paradoxe. Du point de vue juridique, le Brésil est engagé dans la lutte contre la corruption. Il a ratifié toutes les conventions des Nations Unies, de l'OEA et de l'OCDE. En plus, il a harmonisé sa législation pour combattre le manque de probité des fonctionnaires et pour assurer l'égalité d'opportunités dans les marchés publics. Pourtant, le changement est encore lent. A partir des années 70, on perçoit une importante augmentation des affaires de corruption dans tous les niveaux. Quand on parle de la grande corruption, le pays a fait front à des scandales de grande envergure. Mais le système procédural brésilien est complexe et peu efficient, ce qui favorise l'impunité et l'oubli.

Pour avoir une idée de la lenteur des procédures des affaires de corruption, voilà une donnée incontestable : en cinq ans, l'État brésilien a saisi 4 milliards d'euros appartenant à des agents publics corrompus grâce à la coopération judiciaire international. Mais à peine $10 \%$ de cette valeur ont été restitués. Cette constatation est due à plusieurs facteurs : la complexité et la sophistication des opérations financières dans le paradis fiscaux, le blanchiment de capitaux, la possibilité de recourir à plusieurs instances de la justice brésilienne, l'exigence de la chose jugée

mel na ditadura. www.noticias.uol.com.br. 21/01/2014.

35 Les entreprises impliquées dans l'affaire PETROBRAS-BTP: Camargo Corrêa, Odebrecht, Mendes Júnior, Queiroz Galvão, OAS, Engevix, Iesa et Galvão Engenharia.

36 Paulo A Paranaguá. Retentissant scandale de corruption au Brésil. Le Monde du 3 décembre 2014.

37 Letícia Fernandes Gabriela Allegro. Empreiteiras investigadas doaram a partidos R\$ 484,4 milhões nas eleições. www.oglobo.globo.com. 19/01/2015. 
pour exproprier les biens obtenus frauduleusement ${ }^{38}$. Mais il ne faut pas se tromper. Cette augmentation est due plus au renforcement des systèmes de contrôle qu'à l'accroissement de la corruption, puisqu'elle a toujours existé dans le monde politique dans la plus grande opacité.

Mais le Brésil a adopté des mesures importantes dans le cadre de la lutte contre la corruption. En 2012, le Conseil National de Justice, l'organe de contrôle du pouvoir judiciaire, a établi la « Meta 18 », dont l'objectif était de juger les affaires civiles et pénales (manquements au devoir de probité et crimes contre l'administration publique) en cours depuis 2011. Résultat : à la fin 2013, les magistrats avaient jugé 55\%, c'est-à-dire, 63.363 dossiers.

Entre 2003 et 2014, 5.126 fonctionnaires fédéraux ont perdu leurs fonctions publiques à cause de fautes administratives - la majorité en raison de la pratique des actes de corruption (67,17\%) -, selon le rapport du Contrôle général de l'Union publié en $2014^{39}$. Le nombre de punitions est beaucoup plus important, étant donné qu'il n'existe pas d'estimatif par rapport aux peines infligées dans les États membres de la fédération ainsi que dans les municipalités.

Malgré les réponses législatives à l'échelle nationale et internationale, la lutte contre la corruption présente des sanctions à deux vitesses : la sanction électorale et la sanction judiciaire lato sensu. La sanction électorale, autrement dit, le refus des électeurs, ne présuppose nécessairement pas une sanction judiciaire. Il y a encore une certaine tolérance de la société par rapport à certains hommes politiques impliqués dans des scandales financiers. Même devant des preuves irréfutables de participation à des actes de manquement de probité, de nombreux agents publics restent dans leurs fonctions administratives ou sont réélus. Comme les procédures sont longues, complexes et lentes, ils bénéficient de la présomption d'innocence pour continuer à se présenter aux élections en attendant le jugement définitif du pouvoir judiciaire, dont la durée moyenne est de six ans, quelquefois de quinze ans. Cette situation alimente le sentiment d'impunité, d'impuissance, et est fort nuisible à la démocratie.

Le Rapport de l'OCDE sur la corruption transnationale, paru en 2014, signale la gravité de la situation. D'abord la difficulté de la détecter : «De par sa nature même, la corruption internationale est une infraction complexe et occulte. Les schémas de corruption font généralement intervenir une série de transactions extraterritoriales, une multitude d'intermédiaires et des structures d'entreprises complexes. Les difficultés pour détecter les affaires de corruption transnationales

38 Leandro Prazeres. Em quase 5 anos, governo localiza R\$12,4 bi desviados, mas só recupera 10\%. UOL Notícias: www.notícias.uol.com.br. Em 26/11/2014.

39 Cf. Relatório de acompanhamento das punições e expulsivas aplicadas a estatutários no âmbito da administração pública federal. 2014. Mimeo. 
sont donc l'un des obstacles majeurs à une mise en œuvre efficace de la législation anti-corruption ${ }^{40} »$. La recherche de l'OCDE montre que $31 \%$ des affaires ont portées l'attention des autorités répressives à partir du signalement spontané des entreprises privées (audit interne, diligences dans le cadre de fusions-acquisitions, donneur d'alerte, etc.). Tandis que seulement $13 \%$ des affaires ont commencé par l'initiative des autorités chargées de la répression de la criminalité financière. Cette donnée renforce la thèse selon laquelle la faible effectivité des lois, faute de moyens de détection, est la principale raison de la montée de la grande corruption à l'échelle mondiale.

La passation des marchés publics reste encore la principale source de la grande corruption. Selon le rapport de l'OCDE, 57\% des affaires qui ont impliqué le paiement de pots-de-vin à des agents publics étrangers ont eu lieu dans le cadre des appels d'offre. Le plus grave c'est que presque la moitié des affaires de corruption concerne des pays ayant un haut ou un très haut niveau de développement humain. De plus, $60 \%$ des impliqués sont des grandes entreprises, ce qui exige des mécanismes d'autocontrôle pour prévenir des pratiques dommageables à l'équité concurrentielle, voire y renoncer.

\section{REFERENCES}

AEMOUDT, Rudy. Corruption à Foison - Regards sur un phénomène tentaculaire. Paris : L'Harmattan, 2003.

ALLEGRO, Letícia Fernandes Gabriela. Empreiteiras investigadas doaram a partidos $\mathrm{R} \$ 484,4$ milhões nas eleições. www.oglobo.globo. com. Accès: 19/01/2015.

BEZES, Philippe et LASCOUMES, Pierre. Percevoir et juger la corruption politique - enjeux et usages des enquêtes sur les représentations des atteintes à la probité publique. Revue française de Science Politique, vol. 55, n. 5-6, octobre-décembre 2005.

BORRICAND, Jacques. La corruption dans l'entreprise. Morale Économique - des fondements religieux à l'éthique en entreprise. Aixen-Provence : Librairie de l'Université d'Aix-en-Provence, 1999.

BORRICAND, Jacques. Corruption et Politique. In Problèmes Actuels de Science Criminelle, n. IX. Aix-En-Provence : Presses Universitaires d'Aix-Marseille, 1996.

BUEB, Jean-Pierre. La lutte contre la fraude et la corruption dans les marchés publics. Forum Mondial de l'OCDE sur la gouvernance -

40 Rapport de l'OCDE sur la corruption transnationale : Une analyse de l'infraction de corruption d'agents publics étrangers. France : Éditions OCDE, 2014. 
partager les enseignements de la promotion de la bonne gouvernance et de l'intégrité dans le marchés publics. 2006.

CASTRESANA FERNANDEZ, Carlos. Rapport Général. La corruption dans les marchés publics. Allemagne : Edition du Conseil de l'Europe, 1998.

CARVALHO, Getúlio. Da Contravenção à Cleptocracia. Sociologia da Corrupção. Rio de Janeiro: Jorge Zahar, 1987.

DOMMEL, Daniel. Face à la corruption : peut-on l'accepter ? Peut-on la prévenir? Peut-on la combattre ? Paris : Karthala, s/d.

HENRI, Brigitte. Le secteur financier : un partenaire important dans la lutte contre la corruption. Revue Nouvelles Études Pénales, n. 25, 2014.

LASCOUMES, Pierre. Une démocratie corruptible - arrangements, favoritisme et conflits d'intérêts. Paris : Seuil, 2011.

LASCOUMES, Pierre. Corruptions. Paris : Presses de Sciences Po, 1999.

LELIEUR, Juliette et PIETH, Mark . Dix ans d'application de la convention OCDE contre la corruption transnationale. Reccueil Dalloz. Paris, 2008.

MADEIRO, Carlos. Casamento de empreiteiras com poder começou com JK e teve sua lua de mel na ditadura. www.noticias.uol.com.br. 21/01/2014.

MILLET-EINBINDER, Martine. La non-déductibilité des pots-de-vin versés à des agents publics étrangers. Affairisme : la fin du système Comment combattre la corruption. Paris : OCDE, 2000.

MORAIS FILHO, Antônio Evaristo de. O Círculo Vicioso da Corrupção. Sociologia da Corrupção. Rio de Janeiro: Jorge Zahar, 1987.

OCDE. Rapport de 1'OCDE sur la corruption transnationale : une analyse de l'infraction de corruption. France : Éditions OCDE, 2014.

OCDE. Rapport de l'OCDE sur la corruption transnationale : Une analyse de l'infraction de corruption d'agents publics étrangers. France : Éditions OCDE, 2014.

PRAZERES, Leandro Prazeres. Em quase 5 anos, governo localiza R\$ 12,4 bi desviados, mas só recupera 10\%. UOL Notícias: www.notícias. uol.com.br. Em 26/11/2014.

ROCCA, Jean-Louis. La corruption. Paris : Syros, 1993.

TRANSPARENCY INTERNATIONAL. Rapport mondial sur la corruption 2009. La corruption et le secteur privé. Cambridge : Cambridge University Press, 2009. 
TRANSPARENCY INTERNATIONAL. Rapport mondial sur la corruption 2005. Corruption dans le secteur de construction et de la reconstruction d'après-guerre. Paris : Economica, 2005.

TRANSPARENCY INTERNATIONAL. Combattre la corruption Enjeux et perspectives. Paris : Karthala, 2000.

WARD, Gene. Le rôle de la divulgation d'informations dans la lutte contre la corruption dans le financement politique. Transparency International. Rapport mondial sur la corruption 2004. La corruption politique : Cambridge University Press, 2004. 